\title{
Differential proteomic analysis of fetal and geriatric lumbar nucleus pulposus: immunoinflammation and age-related intervertebral disc degeneration
}

Chensheng Qiu ${ }^{1,2,3 \dagger}$, Xiaolin $\mathrm{Wu}^{1 \dagger}$, Jiang Bian ${ }^{4}$, Xuexiao $\mathrm{Ma}^{2}$, Guoqing Zhang ${ }^{2}$, Zhu Guo ${ }^{2}$, Yan Wang ${ }^{2}$, Yandong $\mathrm{Ci}^{5}$, Qizun Wang ${ }^{2}$, Hongfei Xiang ${ }^{2^{*}}$ and Bohua Chen ${ }^{2^{*}}$ (D)

\begin{abstract}
Background: Intervertebral disc degeneration (IVDD) is a major cause of low back pain. Although the mechanism of degeneration remains unclear, aging has been recognized as a key risk factor for IVDD. Most studies seeking to identify IVDD-associated molecular alterations in the context of human age-related IVDD have focused only on a limited number of proteins. Differential proteomic analysis is an ideal method for comprehensively screening altered protein profiles and identifying the potential pathways related to pathological processes such as disc degeneration.
\end{abstract}

Methods: In this study, tandem mass tag (TMT) labeling was combined with liquid chromatography-tandem mass spectrometry (LC-MS/MS) for differential proteomic analysis of human fetal and geriatric lumbar disc nucleus pulposus (NP) tissue. Parallel reaction monitoring (PRM) and Western blotting (WB) techniques were used to identify target proteins. Bioinformatic analyses, including Gene Ontology (GO) annotation, domain annotation, pathway annotation, subcellular localization and functional enrichment analyses, were used to interpret the potential significance of the protein alterations in the mechanism of IVDD. Student's t-tests and two-tailed Fisher's exact tests were used for statistical analysis.

Results: Six hundred forty five proteins were significantly upregulated and 748 proteins were downregulated in the geriatric group compared with the fetal group. Twelve proteins were verified to have significant differences in abundance between geriatric and fetal NP tissue; most of these have not been previously identified as being associated with human IVDD. The potential significance of the differentially expressed proteins in age-related IVDD was analyzed from multiple perspectives, especially with regard to the association of the immunoinflammatory response with IVDD.

(Continued on next page)

\footnotetext{
*Correspondence: ymdx2004qd@126.com; bhchenqd@126.com

${ }^{+}$Chensheng Qiu and Xiaolin Wu contributed equally to this work.

2Department of Orthopedic Surgery, Affiliated Hospital of Qingdao University, Qingdao 266000, China

Full list of author information is available at the end of the article
}

(c) The Author(s). 2020 Open Access This article is licensed under a Creative Commons Attribution 4.0 International License, which permits use, sharing, adaptation, distribution and reproduction in any medium or format, as long as you give appropriate credit to the original author(s) and the source, provide a link to the Creative Commons licence, and indicate if changes were made. The images or other third party material in this article are included in the article's Creative Commons licence, unless indicated otherwise in a credit line to the material. If material is not included in the article's Creative Commons licence and your intended use is not permitted by statutory regulation or exceeds the permitted use, you will need to obtain permission directly from the copyright holder. To view a copy of this licence, visit http://creativecommons.org/licenses/by/4.0/ The Creative Commons Public Domain Dedication waiver (http://creativecommons.org/publicdomain/zero/1.0/) applies to the data made available in this article, unless otherwise stated in a credit line to the data. 
(Continued from previous page)

Conclusions: Differential proteomic analysis was used as a comprehensive strategy for elucidating the protein alterations associated with age-related IVDD. The findings of this study will aid in the screening of new biomarkers and molecular targets for the diagnosis and therapy of IVDD. The results may also significantly enhance our understanding of the pathophysiological process and mechanism of age-related IVDD.

Keywords: Intervertebral disc degeneration, Proteomics, Tandem mass tag, Aging, Inflammatory response

\section{Background}

Low back pain (LBP) severely affects human health in the modern world, placing enormous burdens on patients and society [1]; unfortunately, the pathogenesis of LBP is not entirely understood. Intervertebral disc degeneration (IVDD) is a well-known cause of LBP, especially in elderly people $[2,3]$. The pathogenesis of IVDD is complex and diverse, with aging considered to be the most significant risk factor $[4,5]$. Thus, it is critical to understand the pathophysiological changes associated with disc aging in order to develop an effective treatment for age-related IVDD.

IVDD begins in the nucleus pulposus (NP), the core component of the disc [6]. The anatomic and pathophysiological characteristics of NP tissue change rapidly after birth, causing earlier age-related degeneration in intervertebral discs than other tissues [7-13]. It has been reported that IVDD begins at the age of approximately $15 \sim 20$ years, but some recent studies have demonstrated that it may actually begin much earlier, tracing back as early as the infancy stage [10]. Organismal aging and its ensuing pathophysiological changes can be reflected at the protein level. However, previous research on age-related IVDD has focused on a limited number of proteins and pathways. Animal models and human body fluids are generally used to explore the mechanism of IVDD but may not directly reflect the pathophysiological changes that occur in discs. Overall, few studies have evaluated the biological characteristics of intervertebral discs through comprehensive protein profiling, especially in human NP. Proteomics is a discipline that dynamically studies the composition, function and relation of all proteins under specific physiological or pathological conditions from a holistic perspective [14]. Differential proteomic analysis, which focuses on screening and identifying changes by comprehensive protein profiles between different samples, is an ideal approach for assessment of protein alterations. As proteomic technologies have continued to improve, stable isotope labeling, especially tandem mass tag (TMT) labeling, combined with mass spectrometry (MS), has become an important method for protein quantification [15]. Therefore, comprehensive analysis of protein alterations between fetal and geriatric NP via differential proteomic strategy will provide meaningful information that may be helpful in understanding the mechanism of agerelated IVDD.

In this study, the differentially expressed proteins between fetal and geriatric lumbar disc NP tissues were screened and analyzed by TMT labeling combined with liquid chromatography (LC)-tandem MS (MS/MS). Parallel reaction monitoring (PRM) [16] and Western blotting (WB) techniques were employed to identify target proteins that may be closely related to age-related IVDD. Additionally, bioinformatic analyses, including Gene Ontology (GO) annotation, subcellular localization, domain annotation, pathway annotation, and functional enrichment analyses, were used to interpret the potential significance of the altered protein profiles related to mechanisms of IVDD.

\section{Methods}

\section{Participants and sample collection}

Human fetal lumbar L4/5 intervertebral disc NP samples were collected to form the fetal group $(n=5)$. The specimens were derived from fetuses whose mothers had to terminate their pregnancies due to severe disease or trauma. The fetuses were delivered to the laboratory within $4 \mathrm{~h}$ after induced labor. The gestational ages ranged from 31 to 35 weeks (average $33.2 \pm 1.6$ weeks), which is very close to full term, and the fetuses comprised 2 males and 3 females. L4/5 intervertebral disc NP samples from geriatric patients were collected during spinal fusion for chronic LBP to form the geriatric group $(n=5)$. Magnetic resonance imaging confirmed that the L4/5 intervertebral discs were degenerated but did not exhibit apparent protrusions or sequestrations. The degree of degeneration was Grade IV, as classified by the Pfirrmann grading system [17]. Patients with lumbar disc herniation, lumbar trauma history, spinal tumors, spinal infection, smoking history, diabetes, obesity, and autoimmune diseases were excluded. The geriatric patients ranged in age from 56 to 71 years (average 63.2 \pm 6.2 years) and comprised 3 males and 2 females. The clinical diagnosis was performed by two spine surgeons and a radiologist based on imaging and physical examination and was confirmed during surgery. After separation of the NP using a stereomicroscope, blood and impurities 
on the tissue surface were thoroughly removed by washing with sterile normal saline; the samples were then stored in liquid nitrogen.

\section{Protein extraction}

Each sample was ground to a powder in liquid nitrogen. Four volumes of lysis buffer ( $2 \mathrm{mM}$ EDTA [Sigma, USA], $8 \mathrm{M}$ urea [Sigma], and $1 \%$ Protease Inhibitor Cocktail [Calbiochem, Germany]) were added. The supernatant was collected after ultrasonic processing and centrifugation. A BCA kit was used to determine the protein concentration (Beyotime, China).

\section{Trypsin digestion}

$5 \mathrm{mM}$ dithiothreitol (Sigma) was used to reduce the protein solution at $56^{\circ} \mathrm{C} .30 \mathrm{~min}$ later, at room temperature, $11 \mathrm{mM}$ iodoacetamide (Sigma) was added to alkylate the solution for $15 \mathrm{~min}$ in the dark. Triethylammonium bicarbonate (TEAB) (100 mM) (Sigma) was used to dilute the urea concentration of the protein sample to less than $2 \mathrm{M}$. For the first digestion overnight, trypsin (Promega, USA) was added to the sample (mass ratio, 1:50 trypsin: protein). For the next $4 \mathrm{~h}$, a second digestion was performed (mass ratio, 1:100 trypsin:protein).

\section{TMT labeling}

The peptides were desalted with a Strata X C18 SPE column (Phenomenex, USA) and vacuum freeze-dried after digestion. After being dissolved in 0.5 M TEAB, the peptides were labeled with a TMT kit (Thermo Fisher
Scientific, USA) according to the manufacturer's protocol. Peptides derived from fetal NP samples were labeled with TMT tags of $126,127 \mathrm{~N}, 127 \mathrm{C}, 128 \mathrm{~N}$ and $128 \mathrm{C}$, and geriatric NP samples were labeled with TMT tags of $129 \mathrm{~N}, 129 \mathrm{C}, 130 \mathrm{~N}, 130 \mathrm{C}$ and 131 . The labeled peptides were mixed, desalted and dried.

\section{High performance liquid chromatography (HPLC) fractionation}

The peptide mixtures were fractionated by high-pH reverse-phase HPLC using an Agilent 300Extend-C18 column. In brief, the peptides were first divided into 60 fractions with a linear gradient of acetonitrile (8 to 32\%, $\mathrm{pH}$ 9.0) over $1 \mathrm{~h}$. Then the peptides were combined into 18 fractions and vacuum dried. The detailed fraction sequence is shown in Additional file 1 (HPLC Fraction Sequence).

\section{LC-MS/MS analysis}

Tryptic peptides were dissolved in solvent A (2\% acetonitrile and $0.1 \%$ formic acid) and separated via a gradient of solvent B (90\% acetonitrile and $0.1 \%$ formic acid) as follows: 9 to $25 \%$ (26 $\mathrm{min}$ ), 25 to $38 \%$ (8 $\mathrm{min}$ ), increasing to $80 \%(3 \mathrm{~min})$ and holding at $80 \%$ (3 min). Separation was performed in an EASY-nLC 1000 ultra-performance LC (UPLC) system (Thermo Fisher Scientific). The flow rate was $700 \mathrm{~nL} / \mathrm{min}$. After injection into a nanospray ionization (NSI) source, the peptides were analyzed by MS/MS using an Orbitrap Fusion (Thermo Fisher

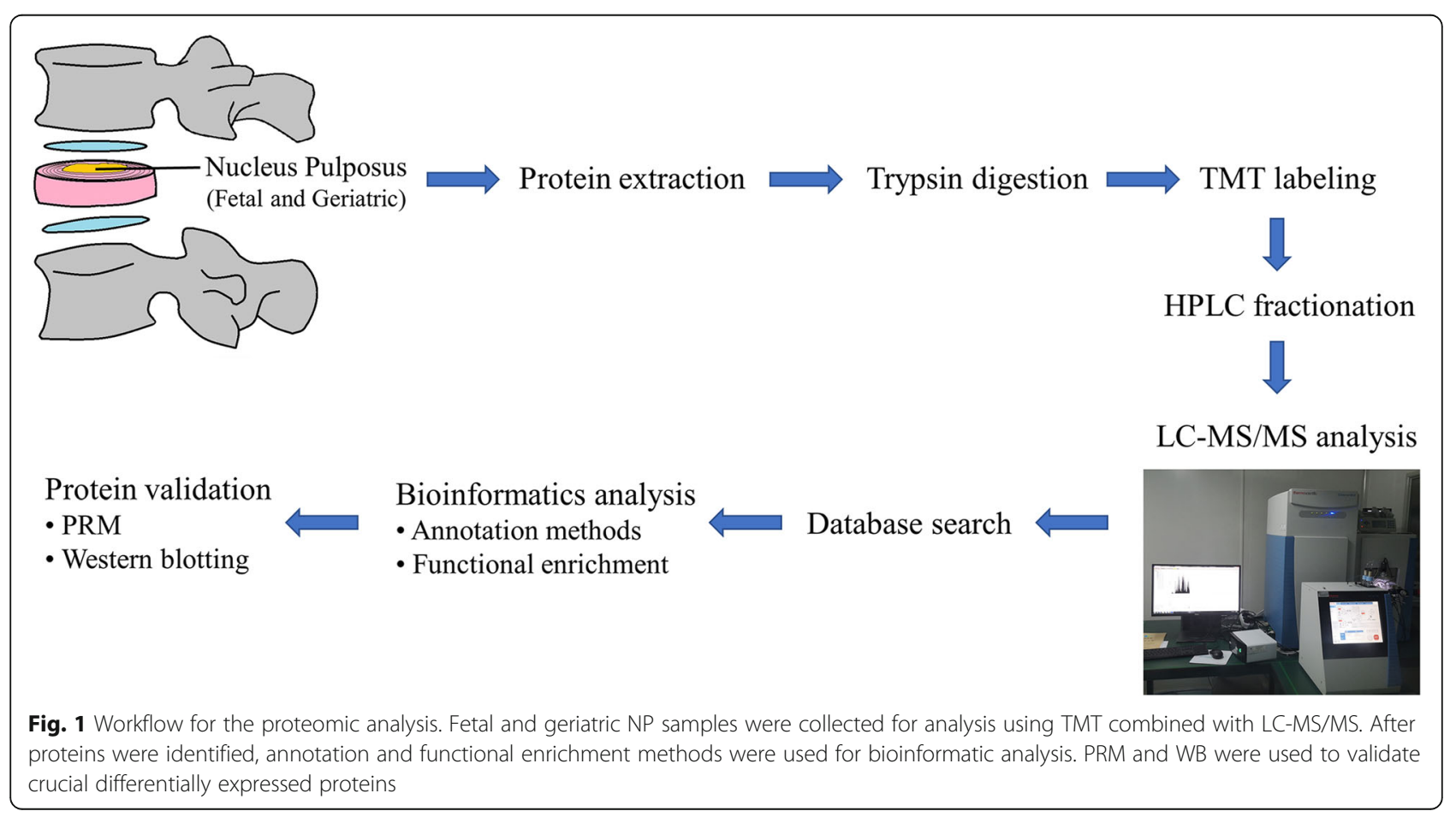


Scientific). The detailed parameter settings are shown in Additional file 1 (LC-MS/MS Analysis Parameters).

\section{Database search}

The raw MS/MS data were processed by MaxQuant search engine (v.1.5.2.8) against the SwissProt human database (20,317 sequences). A contaminant database and a reverse decoy database were combined. The detailed parameter settings are shown in Additional file 1 (Database Search Parameters).

\section{Bioinformatic methods}

GO annotation, domain annotation, pathway annotation and subcellular location analyses were performed. The UniProt-GOA database was used for investigating the GO-annotated proteome, via InterProScan software. The proteins were classified by their GO annotations into three categories: biological process, cellular component, and molecular function. The InterPro database was used to annotate the domains of the identified proteins. The Kyoto Encyclopedia of Genes and Genomes (KEGG) database was employed for protein pathway annotation. WoLF PSORT [18] was used to analyze the subcellular localization. The altered proteins were subjected to functional enrichment (GO, domain, and KEGG pathway) analyses. A two-tailed Fisher's exact test was used for enrichment analysis. A corrected $p$-value of $<0.05$ was considered to indicate significance. The URLs of the databases and software programs noted above are shown in Additional file 1 (Software and Database URLs).

\section{Protein validation by PRM}

The identified proteins were ranked in terms of their degree of change and classified based on their known functions individually. Target proteins were screened for further verification via PRM, a highly targeted MS method, based on their traits and the pathophysiological mechanisms of IVDD. In brief, after being dissolved in solvent A, the tryptic peptides were separated through a gradient of solvent B as follows: 7 to $25 \%$ ( $40 \mathrm{~min}$ ), 25 to $35 \%$ (12 min), increasing to $80 \%(4 \mathrm{~min})$ and holding at $80 \%(4 \mathrm{~min})$. Separation was performed in the EASYnLC 1000 UPLC system. The constant flow rate was $350 \mathrm{~nL} / \mathrm{min}$. The peptides were subjected to NSI source, followed by MS/MS in a Q Exactive ${ }^{\text {Tm }}$ Plus (Thermo Fisher Scientific) coupled online to the UPLC. Each sample was measured separately. The raw data were searched via Skyline (v.3.6) [19]. The detailed parameter settings are shown in Additional file 1 (PRM Analysis Parameters and Skyline Parameters).

\section{Protein validation by WB}

Analysis of the results indicated that the abundance of LTA (protein accession number: P01374) was significantly increased in the geriatric group. We carried out WB to detect the expression of LTA for further verification. In brief, $25 \mu \mathrm{g}$ of protein from each sample was separated by $12 \%$ SDS-PAGE. After transferring the proteins to PVDF membranes (Beyotime), the membranes were subjected to immunoblotting with anti-LTA antibodies (1:1000 dilution, Affinity, USA) at $4{ }^{\circ} \mathrm{C}$ overnight. They were then incubated with HRP-conjugated secondary antibodies (1: 5000 dilution, Proteintech, China) after washing with PBST buffer. Protein expression was detected by enhanced chemiluminescence.

\section{Statistical analysis}

Statistical analysis was performed using SPSS Statistics 22.0. The average value of each identified protein with each group was calculated. Student's t-tests and twotailed Fisher's exact tests were used to evaluate the significance of differences between the two groups. Only differences with a corrected $p$-value of $<0.05$ and a fold change of $>1.50$ or $<0.67(1 / 1.50)$ were considered to indicate differential abundance.

\section{Results \\ Overview of differentially expressed proteins}

The workflow for the proteomic analysis is shown in Fig. 1. The mass error distribution and peptide lengths were used to assess each identified peptide. The prepared samples met the requirements (Additional file 2). Coefficients of variation (CV) [20] was used to evaluate the stability of the data upon examining the variations in abundance among individuals in the same sample group. In the fetal group, $99.3 \%$ of the proteins had CVs of less than 30 , and $92.8 \%$ had CVs of less than $15 \%$. In the geriatric group, $96.4 \%$ of the proteins had CVs of less than 30 , and $77.4 \%$ had CVs of less than $15 \%$. These findings indicated that the differences among the samples in each group were small and that the data were relatively stable (Additional file 3).

In this study, 3259 proteins were identified, among which 2691 were quantified. A quantitative geriatric/fetal ratio of $>1.50$ indicated upregulation; a ratio of $<0.67$ $(1 / 1.50)$ indicated downregulation. Six hundred forty five proteins were upregulated, while 748 were downregulated. The MS information is shown in Additional file 3.

\section{Functional classification of differentially expressed proteins}

\section{GO functional classification}

GO analysis links information between genes and gene products, such as proteins. In this study, the quantified differentially expressed proteins were examined based on their biological properties as identified by $\mathrm{GO}$ analysis. The upregulated and downregulated $\mathrm{GO}$ annotation classifications are shown in Additional file 4. 


\section{Subcellular localization classification}

The subcellular localizations of differentially expressed proteins were predicted and classified by WoLF PSORT software (Fig. 2). The upregulated proteins were concentrated mostly in the extracellular space (291, 47\%), followed by the cytoplasm (109, 18\%) and nucleus (66, $11 \%)$; the downregulated proteins were concentrated mostly in the cytoplasm (291, 39\%), followed by the nucleus $(193,26 \%)$ and extracellular space $(98,13 \%)$ (geriatric/fetal). The proportions of upregulated and downregulated proteins in the mitochondria, plasma membrane, endoplasmic reticulum, cytoplasm/nucleus, peroxisome, cytoskeleton and Golgi apparatus regions were similar.

\section{Enrichment analysis}

Enrichment analysis was performed to detect the functional trends of the altered proteins based on their annotations. The $p$-values obtained in the enrichment test were converted to negative logarithm $(-\log 10)$ values. A larger converted value indicates a more significant enrichment of that functional type.

\section{GO annotation enrichment analysis}

GO annotation enrichment analysis was performed on the subitems in the three level-1 categories. The main results of the GO annotation enrichment analysis are shown in Fig. 3a. In particular, the upregulated proteins (geriatric/fetal) were enriched mainly for terms related to serine-related activity, various types of immune response, complement activation, and defense response; the downregulated proteins were enriched mainly for terms related to RNA biosynthetic process, RNA binding and structural components of the ribosome. These results reflect the activation of immunoinflammatory responses and the decreases in protein synthesis capacity in degenerated discs.

\section{Domain annotation enrichment analysis}

Protein domains are local, compact structural units that cannot be further subdivided and are considered "evolutionary units" of protein sequences. The domain enrichment analysis results are shown in Fig. 3b. In particular, the upregulated proteins (geriatric/fetal) were enriched mainly for immunoglobulin-related and serine proteaserelated domains. These results reflect the activation of the inflammatory response in degenerated discs from a more microscopic perspective.

\section{KEGG pathway enrichment analysis}

The KEGG is an information network of known molecular interactions that includes information on metabolism, genes, cellular processes, and diseases. The results showed that the upregulated proteins (geriatric/fetal) were enriched primarily in the complement and coagulation cascades pathway (hsa04610) and the cytokinecytokine receptor interaction pathway (hsa04060). The downregulated proteins were enriched mainly in the ribosome pathway (hsa03010) (Fig. 4). In the figure, red is used for KEGG pathways enriched for upregulated

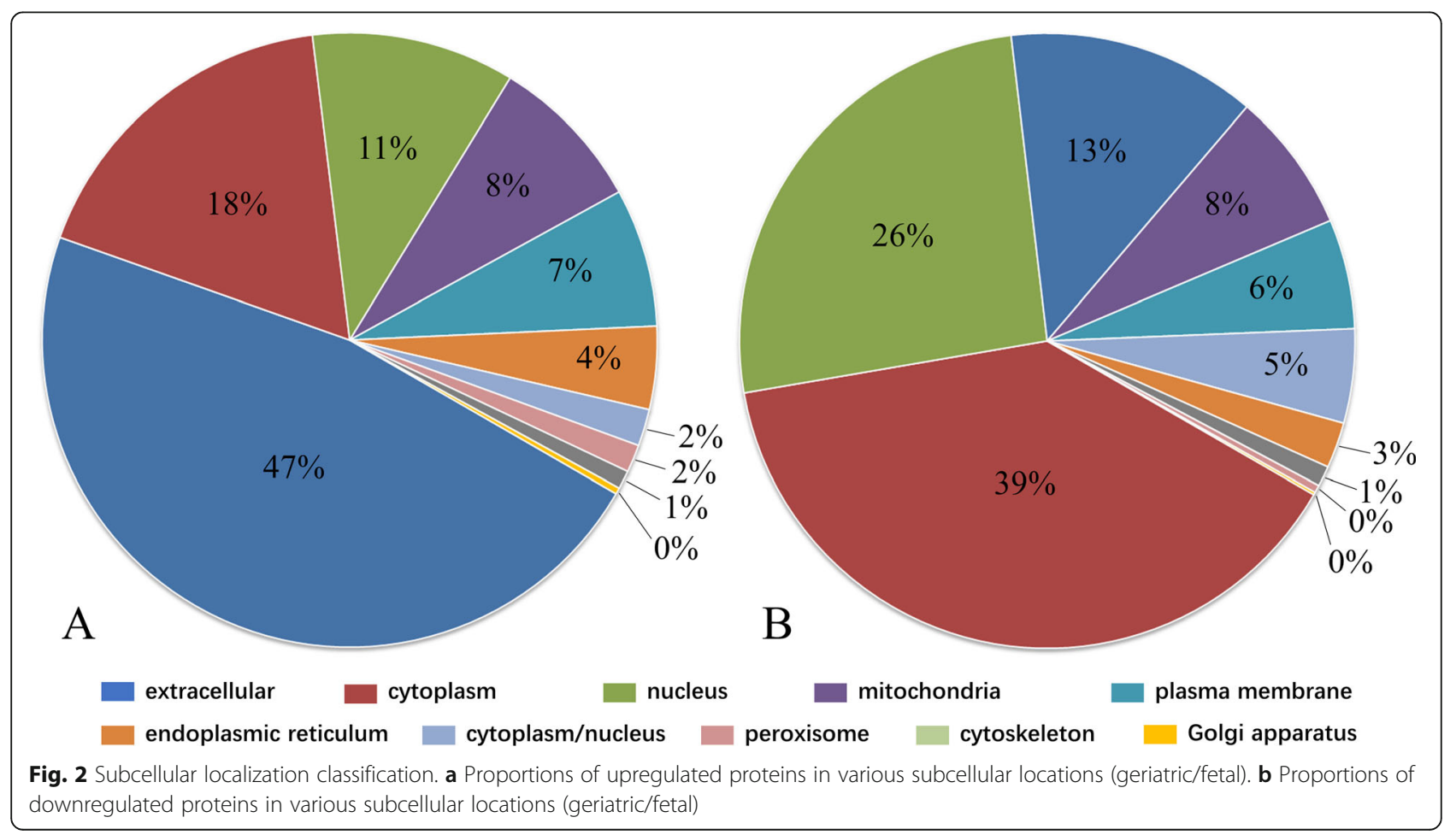




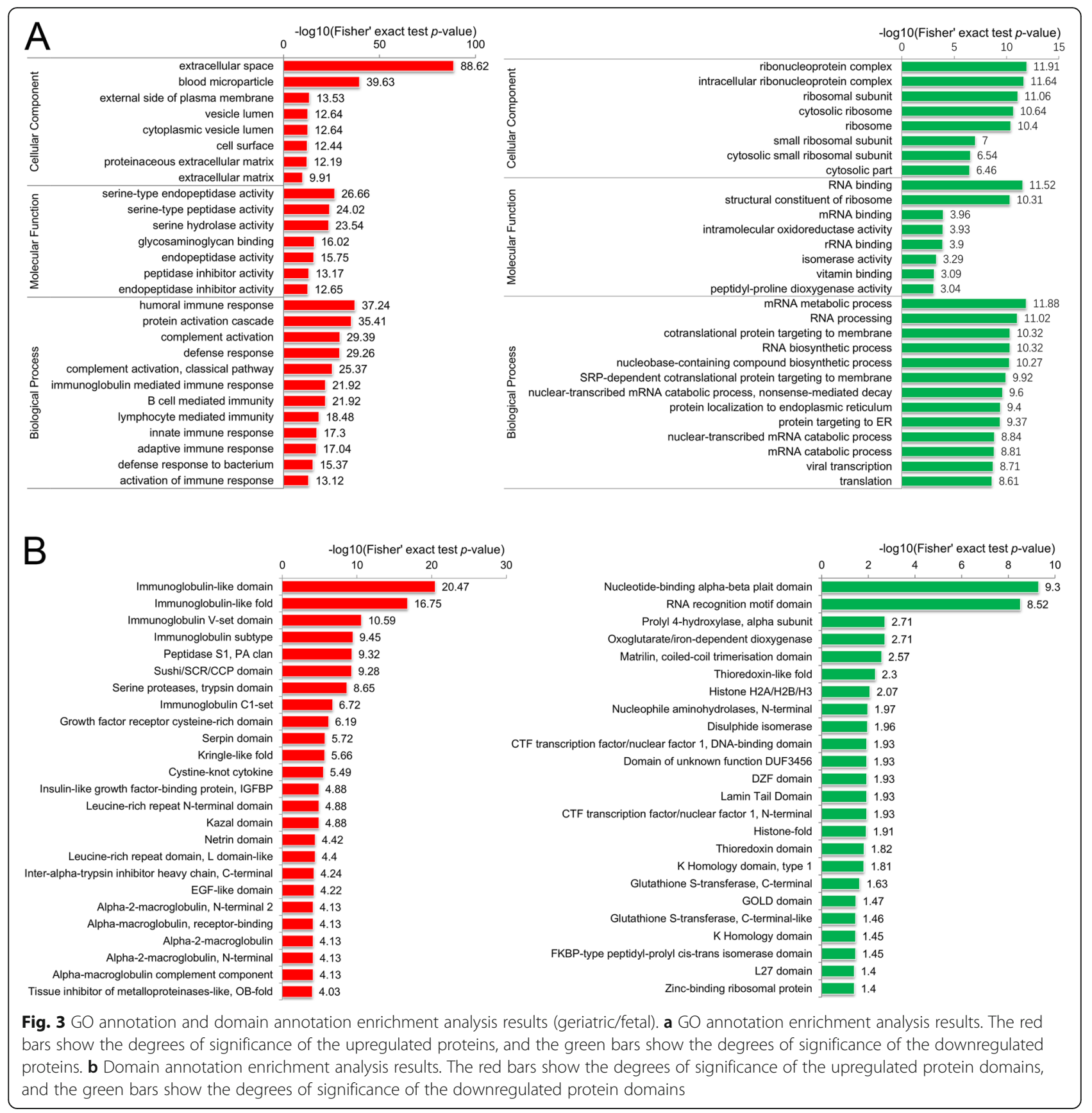

proteins, while green is used for KEGG pathways enriched for downregulated proteins. Among these pathways, complement and coagulation cascades (hsa04610) and cytokine-cytokine receptor interaction (hsa04060) are present, as shown in Fig. 5.

Ribosomes are the main sites of intracellular protein synthesis and are particularly abundant in rapidly proliferating cells. The downregulated proteins (geriatric/fetal) were enriched mainly in the ribosome pathway (hsa03010) (Fig. 4b). In addition, the quantities and proportions of proteins that localized to the cytoplasm and nucleus, involved in the RNA biosynthetic process, and involved in the RNA binding process were reduced (Fig. 2 and Fig. 3a) in degenerated discs. These findings reflect the decreases in protein synthesis ability in aged and degenerated intervertebral discs.

\section{Protein validation by PRM}

Eleven target proteins in human NP tissue that had not previously been clearly reported to be involved in IVDD were screened based on well-known theories and findings related to the pathophysiology of IVDD. PRM was 

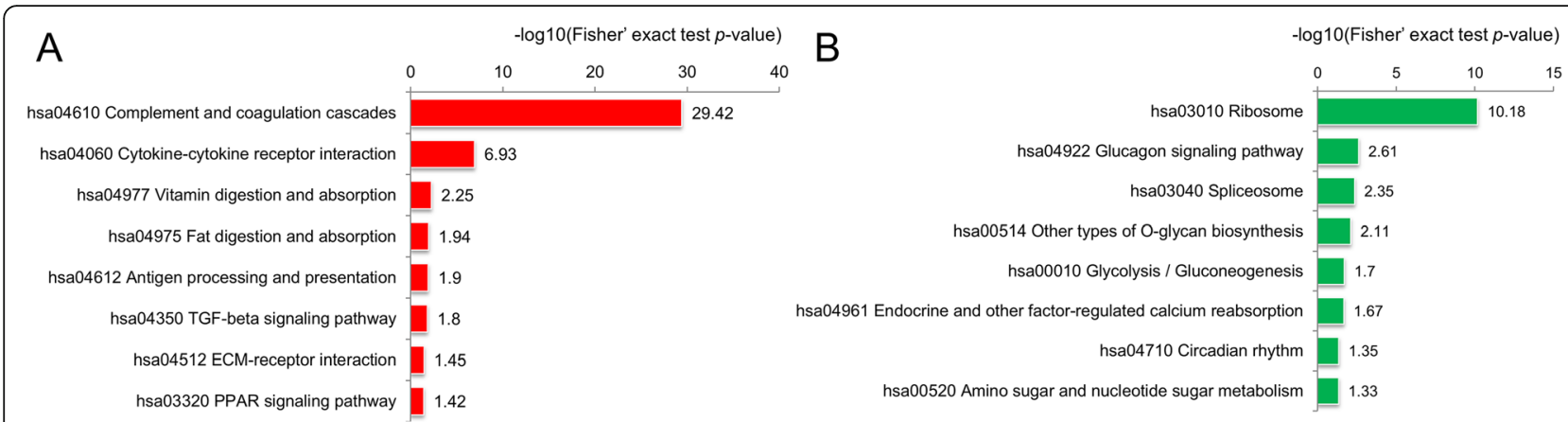

Fig. 4 KEGG pathway enrichment analysis results (geriatric/fetal). a The red bars represent the degrees of significance of upregulated protein enrichment. $\mathbf{b}$ The green bars represent the degree of significance of downregulated protein enrichment

used to further validate the protein alterations, and the main results are provided in Table 1 . The fragment ion peak maps are shown in Additional file 5.

\section{Protein validation by WB}

After conducting PRM, we identified another protein, LTA, that may be closely related to IVDD based on the results of TMT labeling. Compared with the fetal group, the geriatric group exhibited significantly higher expression of LTA (ratio: 32.74, $p$-value: 3.07E-06). Therefore, we performed WB for secondary verification. The WB results confirmed that the expression of LTA was significantly increased in the geriatric group, consistent with the quantitative proteomic analysis results (Fig. 6).

\section{Discussion}

IVDD is a complex clinical condition induced by numerous factors that can eventually lead to the occurrence and development of degenerative disc disorders. In this study, quantitative differential proteomic analysis was used to systematically screen differentially abundant proteins between human fetal and geriatric lumbar disc NP tissues. Through bioinformatics techniques, the relationships of the differentially expressed proteins to agerelated IVDD, especially regarding immunoinflammatory responses, were examined. We also identified 12 differentially expressed proteins closely related to age-related IVDD in human intervertebral discs, LTA, IL11, HSPA8, MATN3, CLU, MFGE8, TNFAIP6, MXRA5, ECM1, SERPINE1, PDGFC and ST13, most of which are reported here for the first time.

The immunoinflammatory response plays a critical role in the pathogenic process of age-related IVDD. Activation of autoimmunity and abnormal expression of inflammatory cytokines lead to disc cell death and dysfunction, increased degradation of the extracellular matrix (ECM) and decreased protein synthesis, thus accelerating IVDD [21]. The enrichment analysis in this study revealed that the upregulated proteins (geriatric/ fetal) were closely associated with various types of immune responses, complement activation, and serine protease-related activity. Many cytokines in the cytokine-cytokine receptor interaction pathway that are involved in the inflammatory response were significantly upregulated, indicating that immunoinflammatory responses were activated in aged and degenerated intervertebral discs. Among these upregulated cytokines, LTA and IL11 particularly attracted our attention. The proteomic alterations were further verified by PRM and WB and the potential molecular mechanism related to these findings is currently under investigation by our research group. The present results suggest that the cytokines LTA and IL11 are closely related to the process of IVDD. The cytokine CXCL12 was shown to be upregulated in the degenerated discs in this study, which is consistent with previous research findings and may have potential value in research on intervertebral disc repair [22]. The activated immunoinflammatory response and the changes in cytokines may be related to phenotypic changes in senescent NP cells and autoimmunity; however, their causality in the pathogenesis of age-related IVDD remains unknown.

IVDD is an age-related degenerative disease, and the degree of IVDD gradually increases with age [5]. The number of senescent NP cells in an intervertebral disc is significantly and positively correlated with the degree of degeneration [4]. Disc cells undergo phenotypic changes during aging and senescence. The proinflammatory phenotype of senescent cells is defined as a senescenceassociated secretory phenotype (SASP). Although the overall protein synthesis ability is decreased in senescent cells, inflammatory cytokines are abnormally overexpressed. Previous studies have demonstrated that these inflammatory cytokines play important roles in deteriorating the disc microenvironment and eventually accelerate IVDD, thus creating a vicious cycle [23].

Intervertebral discs are immunologically privileged tissues [7]. After birth, the NP is isolated from the blood circulation and becomes the largest avascular area; therefore, the NP is immune-privileged throughout life. 


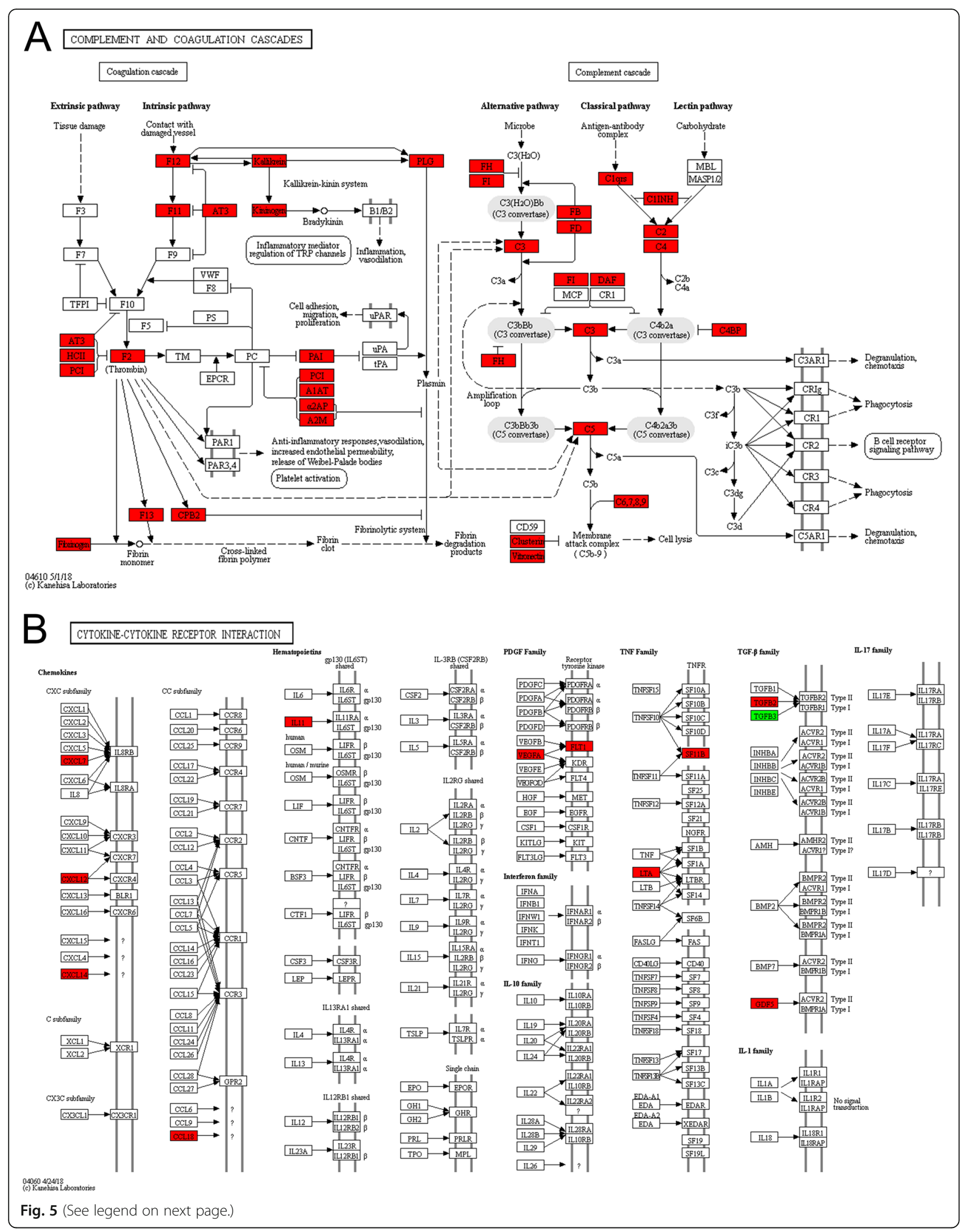


(See figure on previous page.)

Fig. 5 Complement and coagulation cascade pathways (hsa04610) and the cytokine-cytokine receptor interaction pathway (hsa04060) (geriatric/ fetal). a In the coagulation cascade pathway, coagulation factors, including FII, FXI, FXII, and FXIII, and anticoagulation factors, including AT3, HCII, and $\mathrm{PCl}$, were significantly upregulated. In particular, the intrinsic coagulation pathway was activated. Regarding the complement cascade pathway, C1-C9, FB, FH, FD, and FI were significantly upregulated. Both the classic and alternative pathways of the complement cascade were activated. $\mathbf{b}$ In the cytokine-cytokine receptor interaction pathway, the following proteins were significantly upregulated: CXCL7, CXCL12, CXCL14, and CCL18 in the chemokine family; IL-11 in the hematopoietin family; VEGFA in the PDGF family; and LTA in the TNF family. TGFB2 and GDF5 in the TGF- $\beta$ family were also upregulated, whereas TGFB3 was downregulated

This unique anatomical feature renders NP tissue autoantigenic. NP has been suggested to trigger an autoimmune response if the immune-privileged state of the disc is destroyed and the NP is exposed to the immune system [7]; in such case, immune cells and inflammatory cytokines subsequently induce NP cell death and ECM degradation, which may cause or accelerate IVDD. Notably, notochord-secreted molecules restrict axons and/or blood vessels from growing into improper areas [24]; however, this ability gradually diminishes with disc aging. Studies have shown that vascularized granulation tissue can extend from the outer annulus fibrosus into the NP in degenerated discs [25, 26]. Our study also supports this concept. For example, the intrinsic coagulation pathway, which is usually activated due to damage to vessel walls, was significantly activated in degenerated NP tissue. Additionally, vascular endothelial growth factor A (VEGFA), which plays important roles in angiogenesis, vasculogenesis and endothelial cell growth, was upregulated (geriatric /fetal). Given our results and those of previous studies [24-26], we speculate that new blood vessels can grow into deep NP tissue during disc aging and degeneration and that the destruction and reconstruction of blood vessels can lead the antigenic components in NP tissue to be exposed to the circulatory system. As a result, the antigenic components from damaged NP tissue stimulate immunoinflammatory responses when interacting with immune cells, ultimately leading to early IVDD. The alterations of several cytokines in peripheral blood of patients with degenerative disc diseases are consistent with those found in intervertebral discs, such as interleukin-6 and CCL5 [21, 27, 28]. Vascularization of degenerative discs may be a cause of the local inflammatory reaction, which is reflected in the blood circulation system. Therefore, new blood biomarkers found through further studies on the relationship between cytokines in peripheral blood and discs will have potential significance on the early diagnosis and monitoring of IVDD progression. Taken together, the proteomic results from this study suggest a possible pathological condition in which aging and degeneration of the disc may result in vascular growth into the deeper disc.

Ten additional altered proteins were identified: HSPA8, MATN3, CLU, MFGE8, TNFAIP6, MXRA5, ECM1, SERPINE1, PDGFC and ST13. Most of these proteins have not been previously identified to be associated with IVDD in human. These proteins are involved in cell proliferation, cell migration, inflammatory responses, vascular proliferation, fibrosis, ECM degradation, autophagy, apoptosis, cell senescence, tissue remodeling, and other biological processes closely related

Table 1 Protein Quantification by PRM (Geriatric/Fetal)

\begin{tabular}{llllll}
\hline Protein Accession & Protein Name & Gene Name & Ratio (PRM) & $p$-value (PRM) & Ratio (TMT) \\
\hline P20809 & Interleukin-11 & IL11 & +infinite & $7.98 E-11$ & 31.05 \\
P11142 & Heat shock cognate 71 kDa protein & HSPA8 & 0.17 & $5.30 \mathrm{E}-06$ & 0.75 \\
O15232 & Matrilin-3 & MATN3 & 0.01 & $5.01 \mathrm{E}-09$ & 0.38 \\
P10909 & Clusterin & CLU & 35.76 & $4.08 \mathrm{E}-08$ & 16.73 \\
Q08431 & Lactadherin & MFGE8 & 1.27 & $1.60 \mathrm{E}-02$ & 2.14 \\
P98066 & Tumor necrosis factor-inducible gene 6 protein & TNFAIP6 & 78.01 & $9.57 \mathrm{E}-12$ & 14.13 \\
Q9NR99 & Matrix-remodeling-associated protein 5 & MXRA5 & 12.29 & $7.39 \mathrm{E}-11$ & 5.88 \\
Q16610 & Extracellular matrix protein 1 & ECM1 & 4.08 & $8.51 \mathrm{E}-12$ & 5.53 \\
P05121 & Plasminogen activator inhibitor 1 & SERPINE1 & 176.51 & $1.85 \mathrm{E}-07$ & 12.06 \\
Q9NRA1 & Platelet-derived growth factor C & PDGFC & 23.61 & $2.95 \mathrm{E}-09$ & 22.42 \\
P50502 & Hsc70-interacting protein & ST13 & 0.14 & $8.67 \mathrm{E}-08$ & 0.54 \\
\hline
\end{tabular}


A

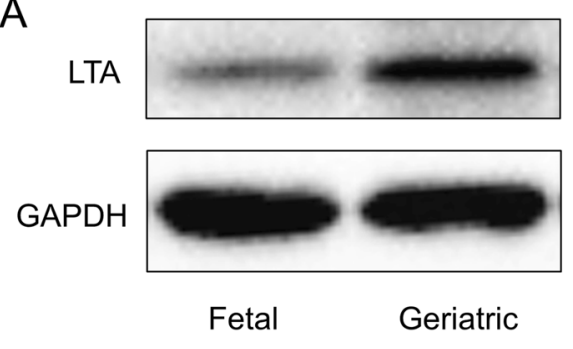

B $22 \mathrm{kDa}$ $36 \mathrm{kDa}$

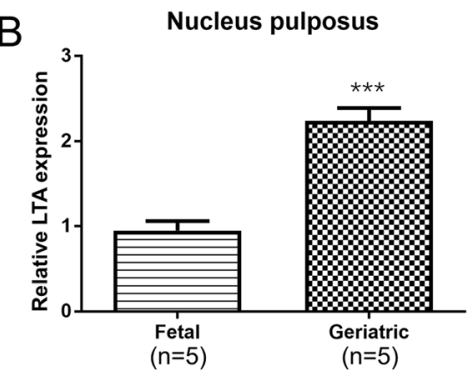

Fig. 6 Protein validation by WB. a Protein lysates from the NP samples of fetuses and geriatric patients were probed with anti-LTA ( $22 \mathrm{kDa}$ ) and anti-GAPDH $(36 \mathrm{kDa})$ antibodies. The level of LTA in the geriatric group was significantly higher than those in the fetal group. $\mathbf{b}$ Results of quantitative analysis of LTA protein levels shown in "A". All data are displayed as the mean $\pm S D$, ${ }^{* * *} p<0.001$

to IVDD [29-37]. The potential molecular mechanism of the involvement of HSAP8 and MATN3 in IVDD has been investigated by our research group, and some of the research findings have already been published [38].

The lack of an absolutely healthy disc source is the most important restrictive factor in basic research on IVDD. Relatively healthy scoliotic/traumatic disc specimens obtained from young patients have often been selected as normal control specimens in previous studies $[39,40]$. However, an increasing number of studies have shown that even these disc specimens have pathological changes, with some exhibiting early degeneration [41, 42]. Other studies have shown that discs in full-term or near-full-term fetuses are very similar to healthy discs in young people and may be selected as ideal healthy control specimens $[43,44]$. Nevertheless, the influences of disc growth and developmental factors on experimental results should not be ignored. As mentioned earlier, recent research progress has demonstrated that degeneration of intervertebral discs may actually occur much earlier than previously reported, tracing back as early as the infancy stage [10]. Therefore, even with the limitations above, our differential proteomic analysis between human fetal and geriatric NP tissues still has farreaching implications for understanding disc aging and degeneration. Despite the small sample size and individual variance in this study, the potential biological targets identified are clinically informative especially because these specimens were from humans. Further investigations will use the database generated by this study to explore the potential mechanisms of age-related IVDD and their significance.

\section{Conclusion}

In conclusion, our differential proteomic analysis comprehensively profiled protein alterations that are potentially associated with age-related IVDD. The results provide a useful database for further investigations into the pathophysiological processes and molecular biological mechanisms of age-related IVDD, especially regarding the association of the immunoinflammatory response with IVDD. New potential biomarkers and molecular targets for the diagnosis and therapy of IVDD have been identified.

\section{Supplementary information}

Supplementary information accompanies this paper at https://doi.org/10. 1186/s12891-020-03329-8.

Additional file 1. Parameter Settings. Detailed parameter settings for the HPLC fraction sequence, LC-MS/MS analysis, database search, PRM analysis, Skyline analysis and database URLS.

Additional file 2. Quality control plots. The mass error and length distributions of peptides.

Additional file 3. Information identified by MS.

Additional file 4. GO annotation classification.

Additional file 5. PRM fragment ion peak maps.

\section{Abbreviations}

IVDD: Intervertebral disc degeneration; TMT: Tandem mass tag; LC: Liquid chromatography; LBP: Low back pain; NP: Nucleus pulposus;

ECM: Extracellular matrix; PRM: Parallel reaction monitoring; WB: Western blotting; GO: Gene Ontology; TEAB: Triethylammonium bicarbonate; HPLC: High performance liquid chromatography; UPLC: Ultra-performance liquid chromatography; MS/MS: Tandem mass spectrometry; KEGG: Kyoto Encyclopedia of Genes and Genomes; CV: Coefficients of variation; VEGFA: Vascular endothelial growth factor A; SASP: Senescence-associated secretory phenotype; MS: Mass spectrometry; NSI: Nanospray ionization

\section{Acknowledgments}

The authors would like to give special thanks to Hao Wang for useful suggestions regarding the revised manuscript; to PTM-Biolabs Co., Ltd. (Hangzhou, China) for the mass spectrometry analysis; and to the editors at American Journal Experts and Yingying Ezell of Medical College of Wisconsin for language editing.

\section{Authors' contributions}

CSQ, XLW and JB performed the experiments and wrote the manuscript. CSQ was a major contributor in writing and interpreting the manuscript. XXM and BHC contributed to the conception and design of the study. YW, YDC, GQZ, and QZW collected patient samples and performed parts of the experiments. ZG and HFX performed the data analysis. The authors read and approved the final manuscript. 


\section{Funding}

This work was supported by Shandong Medical and Health Technology Development Plan Project [2017WS033]; National Natural Science Foundation of China [81802190, 81772412]; Youth Fund Project of the Affiliated Hospital of Qingdao University [2017]; Shandong Provincial Science Foundation, China [ZR2019BH084]; Postdoctoral Science Foundation of China [2019 M652329]; Taishan Scholars Program [tsqn201909190]; Applied Basic Research Project of Qingdao [19-6-2-51-cg].

\section{Availability of data and materials}

The mass spectrometry proteomics data have been deposited to the ProteomeXchange Consortium (http://proteomecentral.proteomexchange. org) via the iProX partner repository [45] with the dataset identifier PXD015000.

\section{Ethics approval and consent to participate}

The work described was carried out following the Code of Ethics of the World Medical Association (Declaration of Helsinki), and this study was approved and supervised by our institutional ethics committee at Affiliated Hospital of Qingdao University (approval number: QDFY WZ 2018-3-1-02). Written informed consent for inclusion in the study was obtained from all patients.

\section{Consent for publication}

Not applicable.

\section{Competing interests}

The authors declare that they have no competing interests.

\section{Author details}

${ }^{1}$ Medical College of Qingdao University, Qingdao 266000, China.

${ }^{2}$ Department of Orthopedic Surgery, Affiliated Hospital of Qingdao University, Qingdao 266000, China. ${ }^{3}$ Department of Orthopedic Surgery, Qingdao Municipal Hospital (Group), Qingdao 266011, China. ${ }^{4}$ Qingdao Eye Hospital of Shandong First Medical University, Qingdao 266000, China. ${ }^{5}$ The Eighth People's Hospital of Qingdao, Qingdao 266000, China.

Received: 28 February 2020 Accepted: 5 May 2020

Published online: 02 June 2020

\section{References}

1. Geurts JW, Willems PC, Kallewaard JW, van Kleef M, Dirksen C. The impact of chronic Discogenic low Back pain: costs and Patients' burden. Pain Res Manag. 2018;2018:4696180.

2. Fujii K, Yamazaki M, Kang JD, Risbud MV, Cho SK, Qureshi SA, et al. Discogenic Back pain: literature review of definition, diagnosis, and treatment. JBMR Pus. 2019;3(5):e10180.

3. Cheung KM, Karppinen J, Chan D, Ho DW, Song YQ, Sham P, et al. Prevalence and pattern of lumbar magnetic resonance imaging changes in a population study of one thousand forty-three individuals. Spine (Phila Pa 1976). 2009;34(9):934-40.

4. Gruber HE, Ingram JA, Norton HJ, Hanley EN Jr. Senescence in cells of the aging and degenerating intervertebral disc: immunolocalization of senescence-associated beta-galactosidase in human and sand rat discs. Spine (Phila Pa 1976). 2007;32(3):321-7.

5. Kim KW, Chung HN, Ha KY, Lee JS, Kim YY. Senescence mechanisms of nucleus pulposus chondrocytes in human intervertebral discs. Spine J. 2009; 9(8):658-66.

6. Haefeli M, Kalberer F, Saegesser D, Nerlich AG, Boos N, Paesold G. The course of macroscopic degeneration in the human lumbar intervertebral disc. Spine (Phila Pa 1976). 2006;31(14):1522-31.

7. Sun Z, Zhang M, Zhao XH, Liu ZH, Gao Y, Samartzis D, et al. Immune cascades in human intervertebral disc: the pros and cons. Int J Clin Exp Pathol. 2013;6(6):1009-14.

8. McCann MR, Tamplin OJ, Rossant J, Seguin CA. Tracing notochord-derived cells using a Noto-cre mouse: implications for intervertebral disc development. Dis Model Mech. 2012;5(1):73-82.

9. Choi KS, Cohn MJ, Harfe BD. Identification of nucleus pulposus precursor cells and notochordal remnants in the mouse: implications for disk degeneration and chordoma formation. Dev Dyn. 2008;237(12):3953-8.
10. Boos N, Weissbach S, Rohrbach H, Weiler C, Spratt KF, Nerlich AG. Classification of age-related changes in lumbar intervertebral discs: 2002 Volvo award in basic science. Spine (Phila Pa 1976). 2002;27(23):2631-44.

11. Aguiar DJ, Johnson SL, Oegema TR. Notochordal cells interact with nucleus pulposus cells: regulation of proteoglycan synthesis. Exp Cell Res. 1999; 246(1):129-37.

12. Roberts S. Disc morphology in health and disease. Biochem Soc Trans. 2002; 30(Pt 6):864-9

13. Alini M, Eisenstein SM, Ito K, Little C, Kettler AA, Masuda K, et al. Are animal models useful for studying human disc disorders/degeneration? Eur Spine J. 2008;17(1):2-19.

14. Wilkins M. Proteomics data mining. Expert Rev Proteomics. 2009;6(6):599603.

15. Thompson A, Schäfer J, Kuhn K, Kienle S, Schwarz J, Schmidt G, et al. Tandem mass tags: a novel quantification strategy for comparative analysis of complex protein mixtures by MS/MS. Anal Chem. 2003;75(8): 1895-904.

16. Peterson AC, Russell JD, Bailey DJ, Westphall MS, Coon JJ. Parallel reaction monitoring for high resolution and high mass accuracy quantitative, targeted proteomics. Mol Cell Proteomics. 2012;11(11):1475-88.

17. Pfirrmann CW, Metzdorf A, Zanetti M, Hodler J, Boos N. Magnetic resonance classification of lumbar intervertebral disc degeneration. Spine (Phila Pa 1976). 2001;26(17):1873-8.

18. Horton P, Park KJ, Obayashi T, Fujita N, Harada H, Adams-Collier CJ, et al. WoLF PSORT: protein localization predictor. Nucleic Acids Res. 2007;35(Web Server issue):W585-7.

19. MacLean B, Tomazela DM, Shulman N, Chambers M, Finney GL, Frewen B, et al. Skyline: an open source document editor for creating and analyzing targeted proteomics experiments. Bioinformatics. 2010;26(7):966-8.

20. Everitt B. The Cambridge dictionary of statistics. Cambridge: Cambridge University Press; 1998.

21. Risbud MV, Shapiro IM. Role of cytokines in intervertebral disc degeneration: pain and disc content. Nat Rev Rheumatol. 2014;10(1):44-56.

22. Ying J, Han Z, Pei S, Su L, Ruan D. Effects of stromal cell-derived factor1alpha secreted in degenerative intervertebral disc on activation and recruitment of nucleus pulposus-derived stem cells. Stem Cells Int. 2019; 2019:9147835

23. Feng C, Liu H, Yang M, Zhang Y, Huang B, Zhou Y. Disc cell senescence in intervertebral disc degeneration: causes and molecular pathways. Cell Cycle. 2016;15(13):1674-84

24. McCann MR, Seguin CA. Notochord cells in intervertebral disc development and degeneration. J Dev Biol. 2016;4(1):1-18.

25. Peng B, Hao J, Hou S, Wu W, Jiang D, Fu X, et al. Possible pathogenesis of painful intervertebral disc degeneration. Spine (Phila Pa 1976). 2006;31(5): 560-6.

26. Johnson WE, Evans H, Menage J, Eisenstein SM, El Haj A, Roberts S. Immunohistochemical detection of Schwann cells in innervated and vascularized human intervertebral discs. Spine (Phila Pa 1976). 2001;26(23): 2550-7.

27. Khan AN, Jacobsen HE, Khan J, Filippi CG, Levine M, Lehman RA Jr, et al. Inflammatory biomarkers of low back pain and disc degeneration: a review. Ann N Y Acad Sci. 2017;1410(1):68-84.

28. Gruber HE, Hoelscher GL, Ingram JA, Bethea S, Norton HJ, Hanley EN Jr. Production and expression of RANTES (CCL5) by human disc cells and modulation by IL-1-beta and TNF-alpha in 3D culture. Exp Mol Pathol. 2014; 96(2):133-8

29. Min JL, Meulenbelt I, Riyazi N, Kloppenburg M, Houwing-Duistermaat JJ, Seymour AB, et al. Association of matrilin-3 polymorphisms with spinal disc degeneration and osteoarthritis of the first carpometacarpal joint of the hand. Ann Rheum Dis. 2006;65(8):1060-6.

30. Santilli G, Aronow BJ, Sala A. Essential requirement of apolipoprotein J (clusterin) signaling for IkappaB expression and regulation of NF-kappaB activity. J Biol Chem. 2003;278(40):38214-9.

31. Raymond A, Ensslin MA, Shur BD. SED1/MFG-E8: a bi-motif protein that orchestrates diverse cellular interactions. J Cell Biochem. 2009;106(6):957-66.

32. Lee $T H$, Wisniewski $H G$, Vilcek J. A novel secretory tumor necrosis factorinducible protein (TSG-6) is a member of the family of hyaluronate binding proteins, closely related to the adhesion receptor CD44. J Cell Biol. 1992; 116(2):545-57.

33. Poveda J, Sanz AB, Fernandez-Fernandez B, Carrasco S, Ruiz-Ortega M, Cannata-Ortiz P, et al. MXRA5 is a TGF-beta1-regulated human protein with 
anti-inflammatory and anti-fibrotic properties. J Cell Mol Med. 2017;21(1): 154-64.

34. Fujimoto N, Terlizzi J, Aho S, Brittingham R, Fertala A, Oyama N, et al. Extracellular matrix protein 1 inhibits the activity of matrix metalloproteinase 9 through high-affinity protein/protein interactions. Exp Dermatol. 2006;15(4):300-7.

35. Kortlever RM, Higgins PJ, Bernards R. Plasminogen activator inhibitor-1 is a critical downstream target of p53 in the induction of replicative senescence. Nat Cell Biol. 2006;8(8):877-84.

36. Jinnin M, Ihn H, Mimura Y, Asano Y, Yamane K, Tamaki K. Regulation of fibrogenic/fibrolytic genes by platelet-derived growth factor $C$, a novel growth factor, in human dermal fibroblasts. J Cell Physiol. 2005;202(2):510-7.

37. Bai R, Shi Z, Zhang JW, Li D, Zhu YL, Zheng S. ST13, a proliferation regulator, inhibits growth and migration of colorectal cancer cell lines. J Zhejiang Univ Sci B. 2012:13(11):884-93.

38. Liu Y, Wang HC, Xiang HF, Jin CH, Chen BH. Expression of HSPA8 in nucleus pulposus of lumbar intervertebral disc and its effect on degree of degeneration. Adv Ther. 2020;37(1):390-401.

39. Rodrigues LMR, Oliveira LZ, Silva M, Accardo CM, Giglio ABD, Pinhal M. Inflammatory biomarkers in sera of patients with intervertebral disc degeneration. Einstein (Sao Paulo). 2019;17(4):eAO4637.

40. Suyama K, Sakai D, Hirayama N, Nakamura Y, Matsushita E, Terayama H, et al. Effects of interleukin-17A in nucleus pulposus cells and its smallmolecule inhibitors for intervertebral disc disease. J Cell Mol Med. 2018; 22(11):5539-51.

41. Sitte I, Klosterhuber M, Lindtner RA, Freund MC, Neururer SB, Pfaller K, et al. Morphological changes in the human cervical intervertebral disc post trauma: response to fracture-type and degeneration grade over time. Eur Spine J. 2016;25(1):80-95.

42. Bertram H, Steck E, Zimmerman G, Chen B, Carstens C, Nerlich A, et al. Accelerated intervertebral disc degeneration in scoliosis versus physiological ageing develops against a background of enhanced anabolic gene expression. Biochem Biophys Res Commun. 2006;342(3):963-72.

43. Ming H, Yuanzheng M, Fengshan H, Li D, Mengming F, Luo X, et al. Cells culture of human fetal lumbar disc and its biological identify. J Practical Orthopaed. 2011;17(2):144-7In Chinese. https://doi.org/10.13795/j.cnki.sgkz. 2011.02.014.

44. Widjaja E, Whitby EH, Paley MN, Griffiths PD. Normal fetal lumbar spine on postmortem MR imaging. AJNR Am J Neuroradiol. 2006;27(3):553-9.

45. Ma J, Chen T, Wu S, Yang C, Bai M, Shu K, et al. iProX: an integrated proteome resource. Nucleic Acids Res. 2019;47(D1):D1211-7.

\section{Publisher's Note}

Springer Nature remains neutral with regard to jurisdictional claims in published maps and institutional affiliations.

Ready to submit your research? Choose BMC and benefit from:

- fast, convenient online submission

- thorough peer review by experienced researchers in your field

- rapid publication on acceptance

- support for research data, including large and complex data types

- gold Open Access which fosters wider collaboration and increased citations

- maximum visibility for your research: over $100 \mathrm{M}$ website views per year

At $\mathrm{BMC}$, research is always in progress.

Learn more biomedcentral.com/submissions 